\title{
Abstracts of the scientific meeting of the Association of Clinical Cytogeneticists held at the Institute of Child Health, London, on 2 and 3 May 1984
}

\author{
The nature and extent of XY pairing at meiotic prophase in \\ man \\ ANN C CHANDLEY \\ MRC Clinical and Population Cytogenetics Unit, Western \\ General Hospital, Edinburgh EH4 $2 X U$.
}

Pairing between the $\mathrm{X}$ and $\mathrm{Y}$ chromosomes at early pachytene in man could be more extensive than has previously been supposed. Circumstantial evidence that it can extend along all of $Y p$, across the $Y$ centromere, and into proximal $\mathrm{Yq}$ will also be presented.

Determination of fetal sex using chorionic villous DNA and $X$ and $Y$ chromosome specific probes

C J CHRISTIE, R G ElLES, JULIA TRACEY, AND J J WATERS Department of Medical Genetics, St Mary's Hospital, Hathersage Road, Manchester M13 OJH.

Chorionic villi can te obtained by direct transcervical aspiration at 8 to 10 weeks' gestation and used for analysis of DNA. A simple method consistently yielding 2 to $3 \mu \mathrm{g}$ of DNA per mg of villous material is described. A DNA probe which hybridises to a sequence common to the $\mathrm{X}$ and $\mathrm{Y}$ chromosomes has been used to determine fetal sex. An assay using two $Y$ specific repetitive DNA probes against only $1 \mu \mathrm{g}$ of DNA is also described. This can be used where the biopsy technique yields only small amounts of villous material.

Steroid sulphatase and the spread of $X$ inactivation M CROCKER, JENNIFER NOBLE, AND C PATEL

Department of Medical Genetics, Churchill Hospital, Headington, Oxford $O X 37 \mathrm{LJ}$.

Normal female levels of STS have been found in a female of the karyotype $45, X$,ter $\operatorname{rea}(X ; 15)(\mathrm{p} 22 ; \mathrm{q} 26)$. This indicates that the STS locus has not been deleted. It is difficult therefore to explain why $97 / 100$ lymphocytes show inactivation of the normal $\mathrm{X}$, since $\mathrm{X}$ inactivation would not normally spread beyond the STS locus. These results will be discussed in relation to $X$ inactivation spreading. Results of studies on STS levels in the Djungarian hamster will also be presented, and the status of $\mathrm{X}$ inactivation of STS in mammals generally will be discussed.
An evaluation of 'Magiscan II'

M G DAKER

Paediatric Research Unit, Guy's Hospital Medical School, Guy's Tower, London SE1 9RT.

Plans are now being drawn up for an evaluation of 'Magiscan II', which is to be undertaken on behalf of the DHSS, to examine the potential of interactive automated chromosome analysis in routine diagnostic work. It is to be hoped that this controversial subject will promote an exchange of ideas, which might prove useful in organising the running of the assessment.

The locus for cystic fibrosis

J H EDWARDS, NORA BLACKWELL, AND J JONASSON Department of Medical Genetics, Churchill Hospitalo Headington, Oxford $O X 37 \mathrm{LJ}$.

An informal search for chromosome abnormalities in Mendelian disorders has yielded a case with cystic fibrosis, suggesting that the CF locus might be at the end of chromosome 13 long arm, band q34. Weak evidence supporting this interpretation comes from a further case and two ancillary observations.

Chorionic villi: trials and tribulations!

NINA GREGSON

Wessex Regional Cytogenetics Unit, General Hospital, Salisbury, Wiltshire SP2 7SX.

A clinical trial for assessing the obstetric risks of trophoblast sampling is in progress at Southampton. The cytogenetics technique used for obtaining banded chromosome preparations from the chorionic villi will be described. The various problems encountered in receipt and processing of the specimens and in banding of the preparations will be discussed.

High resolution karyotyping of human metaphase chromosomes by scanning electron microscopy

CHRISTINE J HARRISON* AND ELSPETH M JACK $\dagger$

* Department of Cell Biology and Cytogenetics, Paterson Laboratories, Christie Hospital and Holt Radium Institute, Manchester M20; and †Department of Medical Genetics, St Mary's Hospital, Manchester M13.

A technique has been developed to examine the same $\mathrm{G}$ banded metaphase chromosomes firstly in the light 
microscope (LM) and followed by the scanning electron microscope (SEM). The chromosome structural involvement in $\mathrm{G}$ banding was confirmed by a positional correlation between the LM G bands and the circumferential grooves between the gyres of the quaternary coils of the metaphase chromosomes, observed in the SEM. The examination of slightly extended metaphase chromosomes in the $\mathrm{LM}$ demonstrated that the $\mathrm{G}$ banding pattern corresponded to the Paris nomenclature for metaphase chromosomes. The arrangement of the circumferential grooves of the same chromosomes, observed in the SEM, was shown to relate to the Paris nomenclature for late prophase chromosomes. Therefore, using the SEM, it is possible to demonstrate the details of late prophase banding in metaphase chromosomes. By detailed comparisons between homologous chromosomes this technique has the potential to study minute chromosome aterrations and to locate precisely the breakpoints involved in structural chromosome rearrangements. The value of the technique will be discussed and examples of its application to antenatal diagnosis and oncological cytogenetics will be presented.

Heterochromatin instability and immunodeficiency MAJ A HULTÉN, P LEEDHAM, AND J WARRAL

Regional Cytogenetics Laboratory, East Birmingham Hospital, Birmingham B9 5ST.

In 1977 we discovered a new type of chromosome instability, involving the heterochromatin in a 5 year old boy with common variable immunodeficiency. The cytogenetic characteristics were briefly reported (Hultén, 1978). Since then, two similar cases have been described, one in Italy (Tiepolo et al, 1979) and one in Belgium (Fryns et al, 1981). Therefore there is now no doubt that we are dealing with 'a new chromosome instability syndrome'. This syndrome is of particular interest because the preliminary information indicates that the abnormalities may be restricted to the heterochromatin of lymphocytes. The aberration, which includes fragility and somatic recombination of the heterochromatic region of chromosomes 1,9 , and 16 , was detected independently and by coincidence in the three patients, ascertained because of mental retardation or an unusual facies or both. In this presentation we will discuss the cytogenetic investigations, using distomycin/Dapi staining, in addition to the standard techniques. The relationship between the chromosome instability and immunodeficiency is not yet clear, but there is an intriguing possibility that we could be dealing with a defective regulatory gene system, involving the repetitive DNA sequences of the heterochromatin previously believed to be without function.

Cytogenetic studies in leukaemia with particular reference to bone marrow transplantation (BMT)

SYLVIA D LAWLER

Section of Human Genetics, Institute of Cancer Research, The Royal Marsden Hospital, Fulham Road, London SW3 6JJ.

Cytogenetic studies are made in patients with leukaemia at diagnosis and after induction of remission by chemo- therapy, before bone marrow grafting. Subsequently, cells in blood and bone marrow are monitored by using sex chromosomes and autosomal polymorphisms as markers for distinguishing between cells of donor and recipient origin. If the patient relapses, the karyotype of these cells can be assessed in the light of previous findings. By using this approach, various chimaeric states in blood and bone marrow have been identified and related to the success of the BMT in eradicating the leukaemia.

In vitro fertilisation: a role for the cytogenetics laboratory ALAN MCDERMOTT AND BARBARA RAY

SW Regional Cytogenetics Centre, Southmead Hospital, Bristol BS10 5NB.

As part of a project to develop the method of in vitro fertilisation and embryo transfer for use in the treatment of infertility in hospitals in Bristol, a simple technical regimen was established using existing National Health Service facilities. The project involves collaboration between obstetricians at three hospitals in Bristol and the Regional Cytogenetics Laboratory. Oocytes are collected at laparoscopy by ovarian follicular aspiration and transported to the Cytogenetics Laboratory in tissue culture medium. Preincubation of the oocytes, preparation/ capacitation of the spermatozoa, in vitro fertilisation, and embryo culture up to the time of transfer to the uterus are all carried out in the Cytogenetics Laboratory. Early indications from this project are that a high success rate for embryo production can be achieved with this arrangement. Our preliminary results clearly indicate that expensive, purpose built units for in vitro fertilisation are not necessary, and that involvement of established Cytogenetics Laboratories with their tissue culture resources and required expertise is likely to prove a more efficient use of scarce resources, making the wider availability of IVF in the National Health Service a more feasible proposition than at present.

\section{Meiotic analysis of a complex rearrangement N SADALLAH AND M A HULTÉN \\ Regional Cytogenetics Laboratory, East Birmingham Hospital, Birmingham B9 5ST.}

Mitotic karyotyping of an infertile man detected a reciprocal translocation between chromsomes 4 and 9 . A testicular biopsy specimen was used for investigations of pachytene spreads and diakinesis/first metaphase. This revealed a complex rearrangement involving chromosome 2 , as well as chromosomes 4 and 9.

A case of interchromosomal effect?

JESSIE L WATT AND D A COUZIN

Department of Genetics, University of Aberdeen, Medical School Buildings, Foresterhill, Aberdeen.

Cytogenetic analysis on the proband revealed a female karyotype with a marker X $(46, \mathrm{XXp}+)$. Parental karyotypes were requested in order to aid identification of the 
extra chromosomal material on $\mathrm{Xp}$. The father was apparently normal $(46, \mathrm{XY})$ while the mother was found to carry a paracentric inversion of chromosome 7 $(46, X X$,inv(7)(q22q35)). An attempt is made to explain these unexpected findings and the possible meiotic events are postulated. This rare family is discussed in the light of the limited publications on paracentric inversion in man. It is proposed that while there may be a significant association between paracentric inversion and sex chromosome abnormality, this could also be classified under the broad and poorly understood heading of 'Interchromosomal Effects'.

\section{Correction}

In the article "A screening programme for the prospective prevention of Mediterranean anaemia in Latium: results of seven years' work" by Bianco et al (Journal of Medical Genetics 1984;21: 268-71) the the following corrections should be noted:

On page 270 the last paragraph of the right-hand column from the 9th to 14th line should read: "... should be attained in about 5 more years. In fact, it can be calculated that there are about 800000 young couples of child-bearing age in Latium (that is half of the 1600000 living in the region) and of these 800000 couples approximately 350 , that is $\left(2 \cdot 1 \times 10^{-2}\right)^{2} \times 8 \cdot 10^{5}$, are at risk. If 40 couples at risk...". The last line of the right hand column should read: "Therefore in about 5 more years the 'arreas' will ...".

On page 271 the second line of the left-hand column should read: “... prospective couples have to be identified every year." 